\title{
Synthesis of Scalable Planar Scissor Linkages with Anti-Parallelogram Loops
}

\author{
Ş. Gür ${ }^{1}$, C. Karagö̈ ${ }^{2}$, G. Kiper ${ }^{3}$ and K. Korkmaz ${ }^{4}$ \\ Iİmir Institute of Technology,Turkey,e-mail:sebnemgur@iyte.edu.tr \\ ${ }^{2} \dot{z} z m i r$ Institute of Technology,Turkey, e-mail: cevahirkaragoz@iyte.edu.tr \\ ${ }^{3}$ İzmir Institute of Technology,Turkey, e-mail: gokhankiper@iyte.edu.tr \\ ${ }^{4}$ İzmir Institute of Technology, Turkey, e-mail: koraykorkmaz@iyte.edu.tr
}

\begin{abstract}
.
Scissor linkages are commonly used as mechanisms for scaling objects. They constitute a significant portion of deployable structures. Since 1960s many researchers sought to form novel structures using the scissor units. In 1990s Hoberman brought a new perspective to the field when he used the loops, not the units to form linkages. Starting from mid 2000s, other researchers joined into this new approach of design. One of the latest researches presented a design for scaling a circular forms with antiparallelogram loops. This study shows that an anti-parallelogram loop assembly can also be used for scaling planar curves with variable curvature.
\end{abstract}

Key words: scalable scissor linkage, anti-parallelogram loop, loop assembly method

\section{Introduction}

Mechanisms that can transform between an open (deployed) and a closed (stowed) configuration are called deployable structures [8]. Scissor linkages are widely used for deployable structures. Scissor-like elements (SLEs) are ternary links with revolute joints. Two SLEs connected at the middle joint constitute a scissor unit. The first academic study on SLEs was conducted by Pinero in 1961 for his design of a deployable theater composed of pantographic elements [17]. Escrig defined the foldability conditions $[2,3]$. The subject remains to be of interest today for many other researchers $[13,16,22]$.

A special type of deployable structures is scaling mechanisms. As the name suggests these mechanisms maintain a specific form while they expand and shrink. Hoberman introduced angulated SLEs in 1990 for scaling mechanisms [10, 11]. Endpoints of an angulated SLE follows radial lines during deployment while preserving the angle in between provided that the SLE satisfies certain geometrical 
conditions. In 1997 You and Pellegrino [20] reported the necessary conditions for deployment with subtended angles and introduced Generalized Angulated Elements (GAEs) of Type I and Type II.

Hoberman's invention also introduces a novel approach: assembling loops to synthesize structures. Unlike the commonly used method of assembling scissor units, Hoberman places rhombus loops on a closed curve and determines the link lengths of the angulated SLEs using the edges of the rhombi [9, 10]. Liao and Li [14], Kiper and Söylemez [12] also made use of rhombi for scaling planar graphs and polygons. Yar et al. [19] used kite and dart loops and Gür et al. [5] used antiparallelogram loops to obtain planar linkages comprising SLEs.

\section{Assembly of Loops}

There are several ways to classify scissor units used in scissor linkages. In the most common classification scissor linkages are composed of three types of scissor units: transitional, polar and angulated units. Another classification by Zhang et al. [21] reveals three corresponding types: parallel, symmetric and isogonal units. Basically, these units are used for altering either the linear lengths, or the circular arc length or the curvature of a curve segment. Maden et al. [15] present the alternative assemblies of scissor units and provide analysis and design criteria. When the scissor linkages in the literature are examined, it is seen that rhombus, kite, dart, parallelogram, anti-parallelogram and general convex and concave shaped loops can be found. As an example, a translational linkage with rhombus loops and a polar linkage with kite loops are seen in Fig. 1.

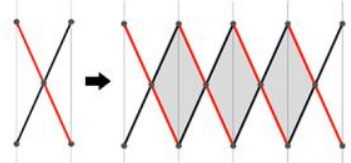

Translational scissor linkage with rhombus loops

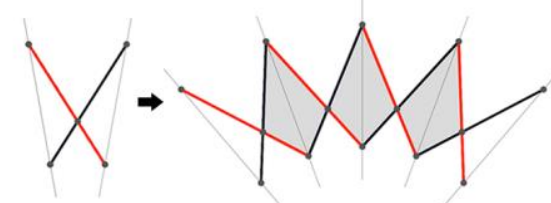

Polar scissor linkage with kite loops

Fig. 1 Loop geometries of two scissor structures produced with primary scissor elements

In order to form a scalable polygon, Hoberman [9] places rhombi on the edges of a polygon, which is usually a segmented approximation of a continuous curve (Fig. 2). In this study, anti-parallelogram loops are used to form scaling linkages. 

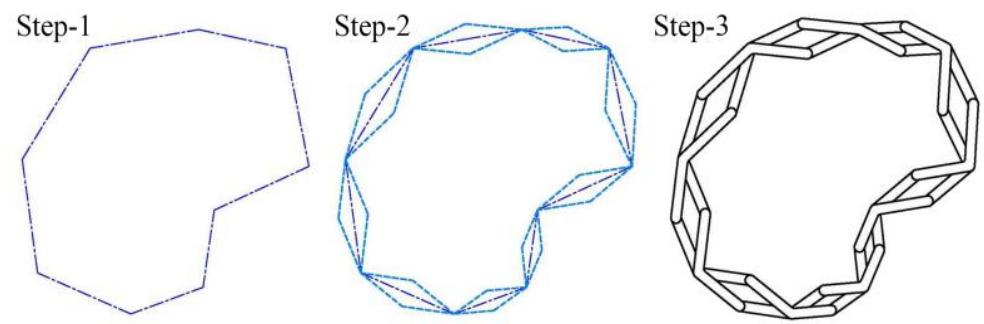

Fig. 2 Loop assembly method as shown in MIT Class 6.S080 by Hoberman [9]

\section{Anti-Parallelogram Loop Assemblies}

Anti-parallelogram, also known as crossed parallelogram or contraparallelogram, has two short and two crossing long edges of equal length. A linkage forming an anti-parallelogram loop can be folded flat in both directions of the motion. Mirror symmetry of the loop about a vertical axis is preserved during deployment. The symmetry axis passes through the crossing point of the long edges (Fig. 3).

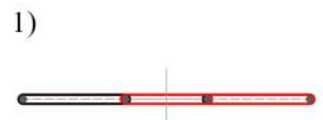

4)

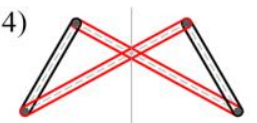

2)

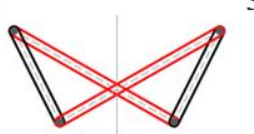

3)

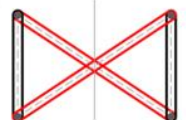

5)

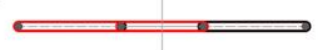

Fig. 3 Motion of anti-parallelogram loop

The loops in an assembly are connected at the corners which symbolize the joint positions. There are many possible alternatives of connecting the loops depending on the choice of connection corner and also their rotation and configuration. The alternatives of anti-parallelogram loop arrays were studied by Gür in detail and a real-life application of one of the linkages resulting from the study was published in 2017 [4, 6]. In another former study of Gür et al. [5] an assembly with an alternating order of anti-parallelogram loops was used to form ring-like structures with radial deployment. This study presents the use of antiparallelogram loops to form linkages for scaling planar curves with variable curvature using the same array type used in [5] for ring-like structures (Fig. 4). SolidWorks software is used to create a kinematic model and visualize the motion. It is observed that the curve form is preserved during the motion (Fig. 5). 


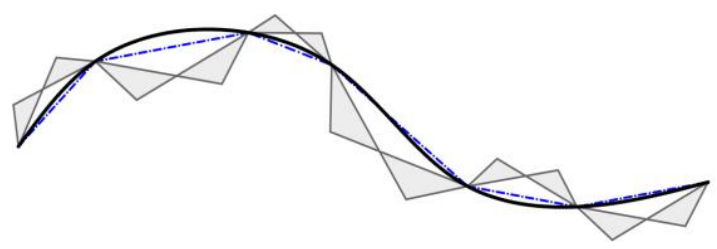

Fig. 4 Anti-parallelogram loop assembly on a polyline with variable curvature

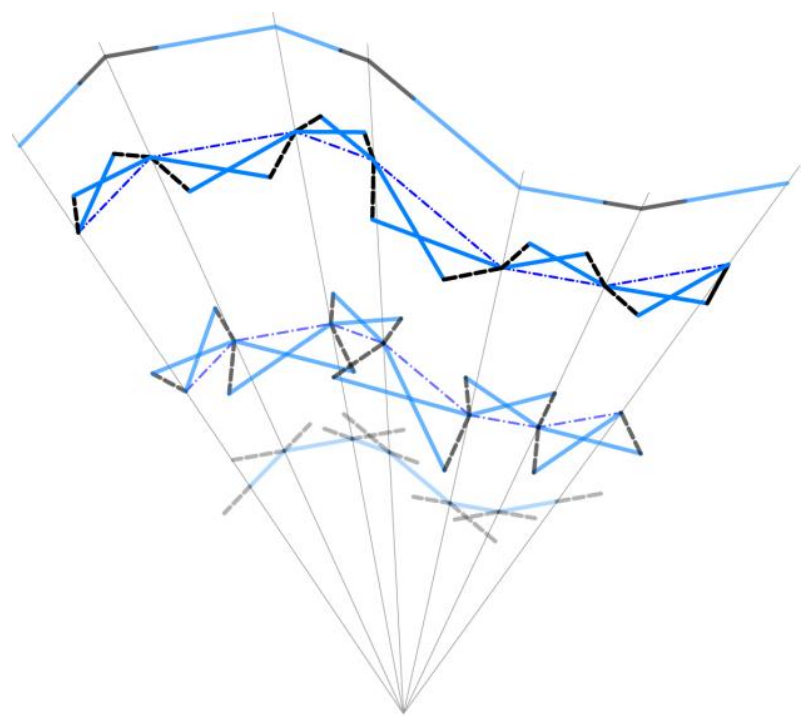

Fig. 5 Deployment of the linkage

When a planar curve is given, it can be discretized at some points to approximate it as a polyline. The discretization points can be selected to be equally spaced on the curve, or the spacing can be decided based on the change in curvature [7] or according to special design criteria of a specific problem. Since the main characteristics of a curve is how it is curved as one moves on the curve, the curvature must be somehow represented with the polyline approximation. This can be done considering the centers of the circles passing through every three consecutive points of the polyline or considering the angle between two consecutive line segments of the polyline (Fig. 6). The center of a circle passing through three points is the intersection of perpendicular bisectors of two pairs of points. Hoberman makes uses of these "normal lines" in [11] for his construction with rhombi. For most scissor units, the normal lines pass through joints, however this is not the general case. In $[1,21]$ unit lines are defined as the lines connecting the joint pairs on the two sides of a scissor unit, but these unit lines do not always coincide with 
normal lines and may not correspond to any geometrical characteristics of the approximated curve.

For an anti-parallelogram, the vertical symmetry axis, which does not pass through any joints but passes through the intersection of the crossing links, corresponds to the normal lines when the loops are assembled on a polyline.

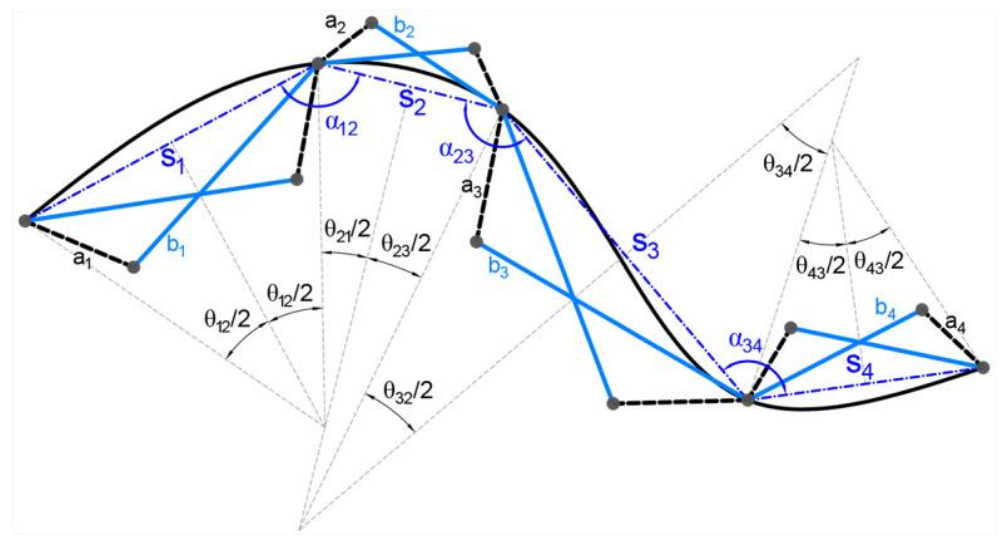

Fig. 6 Parameters of the linkage

Let the line segment lengths of a polyline be represented by $S_{n}$ for segment $n$ The ratio of $\mathrm{n}^{\text {th }}$ segment length to the first one can be determined as $\mathrm{k}_{\mathrm{n}}$ :

$$
\frac{S_{2}}{S_{1}}=k_{2}, \quad \frac{S_{3}}{S_{1}}=k_{3} \quad \cdots \quad \frac{S_{n}}{S_{1}}=k_{n}
$$

The link lengths of the anti-parallelogram loops are determined using the ratio between the short $\left(a_{1}\right)$ and long edge $\left(b_{1}\right)$. The ratio $R$ should be equal for all loops on every segment, hence resulting in GAEs of Type II, i.e. similar GAEs:

$$
\mathrm{R}=\frac{\mathrm{b}_{1}}{\mathrm{a}_{1}}=\frac{\mathrm{b}_{2}}{\mathrm{a}_{2}}=\ldots=\frac{\mathrm{b}_{\mathrm{n}}}{\mathrm{a}_{\mathrm{n}}}
$$

At the fully deployed form, the sum of the link lengths is equal to segment lengths. Therefore, the kink angle $\alpha_{n n+1}$ of an angulated SLE meeting at the vertex of a polyline is simply the angle between the segments meeting at the vertex. The kink angle at a vertex is equal to the summation of halves of subtended angles of the neighboring segments. $\alpha_{n n+1}+\left(\theta_{n n+1}+\theta_{n+1 n}\right) / 2=180^{\circ}$ in Fig. 6. Since the kink angles of a pair of angulated SLEs meeting at a vertex are equal to each other, all anti-parallelogram loops deploy with the same ratio during the motion, hence resulting in a scaling linkage. A more detailed proof can be found in [5]. The remaining link lengths can be found as follows: 


$$
S_{n}=a_{n}+b_{n}=a_{n}(1+R) \Rightarrow a_{n}=\frac{S_{n}}{1+R}, b_{n}=S_{n}-a_{n}
$$

Also the maximum deployed-to-compact form ratio can be found as:

$$
\text { Compactness ratio: } 100 \times \frac{b_{n}-a_{n}}{b_{n}+a_{n}}=100 \times \frac{R-1}{R+1}
$$

The ratio $\mathrm{R}$ and compactness ratio can be utilized as design measures. Once the link lengths are decided, kinematic analysis of the resulting linkage can be performed. Derivation of the kinematic analysis formulations are straightforward (see for ex. [18]) and are not presented here for conciseness. The formulations are implemented in Microsoft Office Excel (Fig. 7). Cells highlighted in blue color are the inputs. The fully deployed and partially deployed forms of a polyline with a closed contour, i.e. a polygon, can be seen in Fig. 8. In Fig. 8, the link lengths ratio is $\mathrm{R}=2.5$, but any ratio can be selected by the designer.

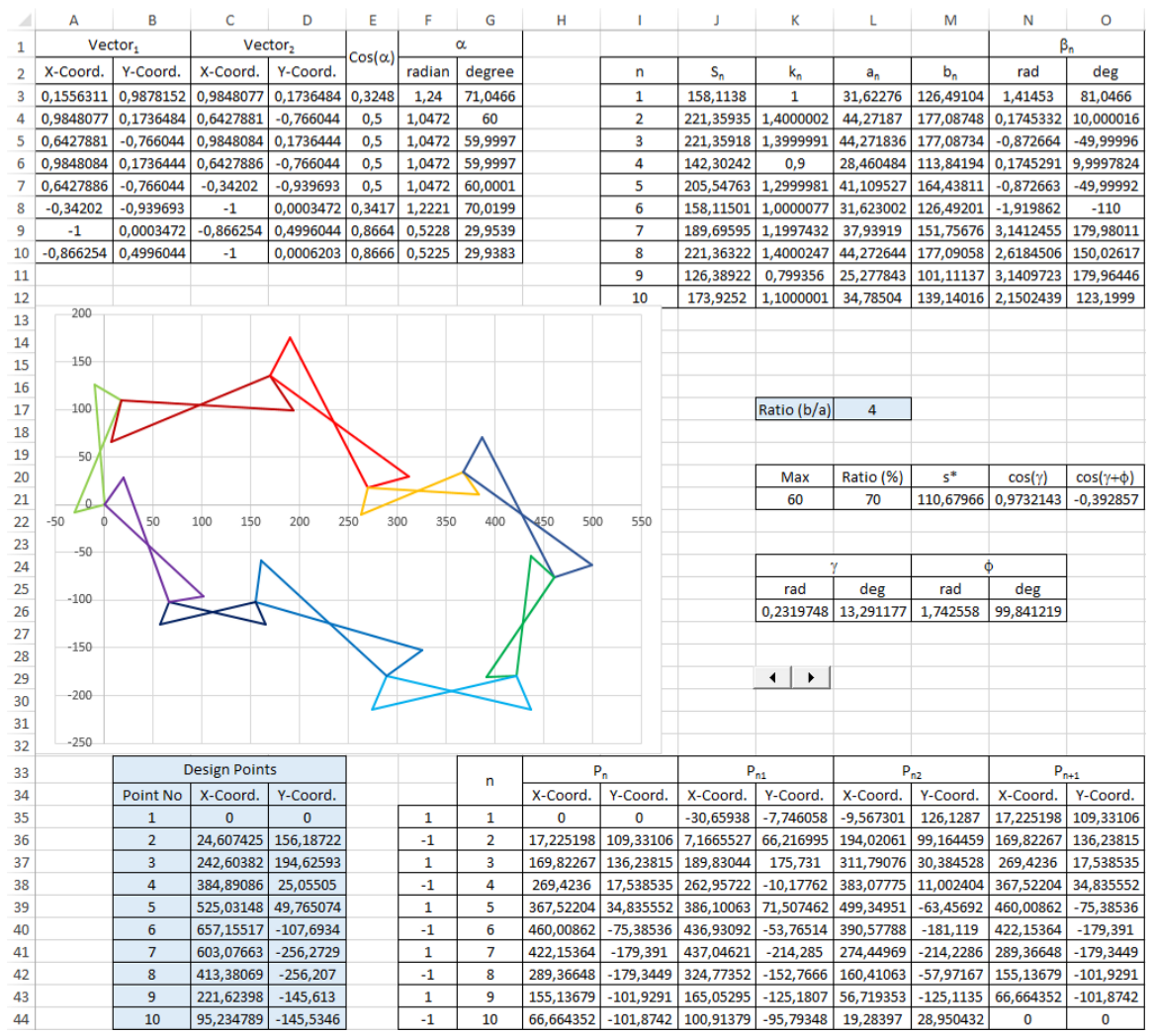

Fig. 7 Kinematic analysis in Excel 


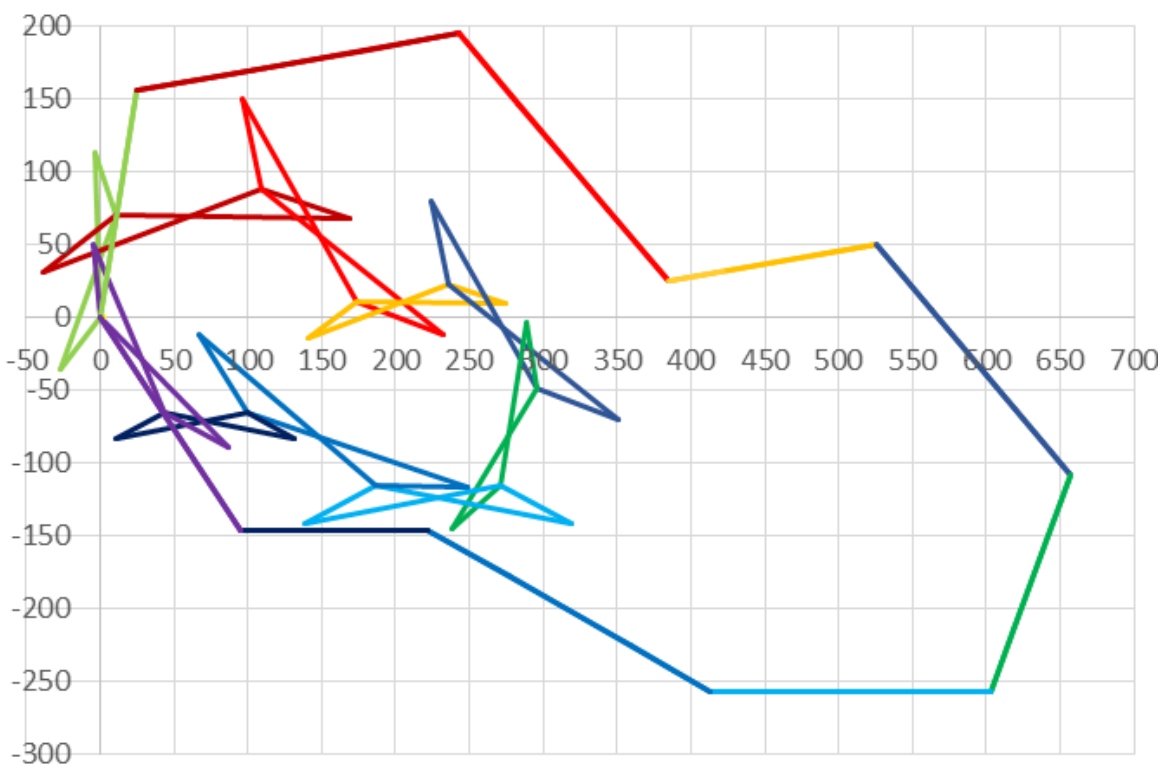

Fig. 8 Deployment of a linkage visualized in Excel graphics

\section{Conclusions}

Previous studies showed that a circular assembly of anti-parallelogram loops in a specific array, whether they are identical or similar, is capable of radial deployment. In this study an assembly of same array type is used for deployable structure that can scale a curve with variable curvature. SolidWorks and Excel softwares are used for modelling and calculation. Once the curve is approximated by a poly-line, there is only one free parameter, ratio $\mathrm{R}$, in order to determine the link lengths of the linkage. It is demonstrated that such a linkage is capable of radial deployment with subtended angles. Along with many other scissor mechanisms, antiparallelogram loop assemblies have potential applications in kinetic architecture.

Acknowledgments This project has received funding from the European Union's Horizon 2020 research and innovation programme under the Marie Skłodowska-Curie grant agreement No 689983.

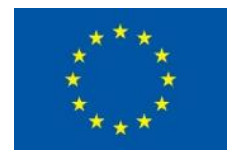




\section{References}

1. De Temmerman, N.: Design and Analysis of Deployable Bar Structures for Mobile Architectural Applications. Ph. D. thesis, Vrije Universiteit Brussel, Brussels, Belgium, (2007)

2. Escrig, F.: Expandable Space Frame Structures. In: Proceedings of the 3rd International Conference on Space Structures, Elsevier Applied Science Publishers, Guildford (1984)

3. Escrig, F.: Expandable space structures. Int. J. Space Struct., 1(2), 79-91 (1985)

4. Gür, Ş.: Design of Single Degree-of-Freedom Planar Linkages with Antiparallelogram Loops Using Loop Assembly Method. MSc Thesis, İzmir Institute of Technology (2017)

5. Gür, S. and Korkmaz, K. and Kiper, G.: Radially expandable ring-like structure with antiparallelogram loops. In: Proceedings of the International Symposium of Mechanism and Machine Science ISMMS-2017, Baku, Azerbaijan (2017)

6. Gür, S. and Yar, M. and Korkmaz, K.: A novel two degrees-of-freedom structural mechanism proposal for multi-functional transformable bridge. J. Fac. Eng. Archit. Gaz., 32(4), 1379$1392(2017)$

7. Hamann, B. and Chen, J.: Data point selection for piecewise linear curve approximation. Comput. Aided Geom. Des., 11(3), 289-301 (1994)

8. Hernández Merchan, C. H.: Deployable Structures. MSc Thesis, Massachusetts Institute of Technology (1987)

9. Hoberman, C. and Demaine, E. and Rus, D.: MIT Class 6.S080 (Aus) - Mechanical Invention through Computation, Mechanism Basics. Available via MIT online courses http://courses.csail.mit.edu/6.S080/ (2013)

10. Hoberman, C.: Reversibly Expandable Doubly-Curved Truss Structure, Patent no: US4942700A (1990)

11. Hoberman, C.: Radial Expansion/Retraction Truss Structures, Patent no: US5024031A (1991)

12. Kiper, G and Söylemez, E.: Irregular polygonal and polyhedral linkages comprising scissor and angulated elements. In: Proceedings of the 1st IFToMM Asian Conference on Mechanism and Machine Science (CD), Taipei (2010)

13. Langbecker, T.: Kinematic analysis of deployable scissor structures. Int. J. Space. Struct., 14(1), 1-15 (1999)

14. Liao, Q. and Li, D.: Mechanisms for scaling planar graphs. Chin. J. Mech. Eng., 8, 26 (2005)

15. Maden, F. and Korkmaz, K. and Akgun, Y.: A review of planar scissor structural mechanisms: geometric principles and design methods. Archit. Sci. Rev., 54(3), 246-257 (2011)

16. Nagaraj, B. P. and Pandiyan, R. and Ghosal, A.: Kinematics of pantograph masts. Mech. Mach. Theory, 44(4), 822-834 (2009)

17. Piñero E. P.: Project for a mobile theatre. Archit. Design, 12(1), 154-155 (1961)

18. Söylemez, E.: Using Computer Spreadsheets in Teaching Mechanisms. In: Proceedings of EUCOMES 08. Springer, pp. 45-53 (2008)

19. Yar, M. and Korkmaz, K. and Kiper, G. and Maden, F. and Akgün, Y and Aktaş, E.: A novel planar scissor structure transforming between concave and convex configurations. Int. J. Comp. Meth. and Exp. Meas., 5(4), 442-450 (2017)

20. You, Z. and Pellegrino, S.: Foldable bar structures. Int. J. Solids Struct., 34(15), 1825-1847 (1997)

21. Zhang, R. and Wang, S. W. and Chen, X. J. and Ding, C. and Jiang, L. and Zhou, J. and Liu, L. G.: Designing planar deployable objects via scissor structures. IEEE T. Vis. Comput. Gr., 22(2), 1051-1062 (2016)

22. Zhao, J. S. and Chu, F. L. and Feng, Z. J.: The mechanism theory and application of deployable structures based on SLE. Mech. Mach. Theory, 44(2), 324-335 (2009) 